\title{
Parâmetros genéticos de características reprodutivas de touros e vacas Gir leiteiro
}

\author{
Mário Luiz Santana Júnior ${ }^{1}$, Paulo Sávio Lopes ${ }^{2}$, Rui da Silva Verneque ${ }^{3}$, Rodrigo Junqueira \\ Pereira $^{1}$, Marcos Rodrigues Lagrotta ${ }^{1}$, Maria Gabriela Campolina Diniz Peixoto ${ }^{3}$
}

1 Programa de Pós-Graduação em Zootecnia, UFV - Viçosa, MG. Bolsista CAPES.
2 Departamento de Zootecnia, UFV - Viçosa, MG.

${ }^{3}$ Embrapa Gado de Leite - Juiz de Fora, MG.

RESUMO - Com o objetivo de estimar parâmetros genéticos para a idade ao primeiro parto, perímetro escrotal e características do sêmen e avaliar a tendência genética da idade ao primeiro parto para animais da raça Gir Leiteira, foram analisadas medidas de 7.055 fêmeas e 97 machos de diversos rebanhos brasileiros. Os componentes de covariância foram estimados utilizando-se o método da máxima verossimilhança restrita, sob modelo animal, em análises unicaracterísticas. O modelo para características do sêmen incluiu os efeitos fixos central-ano-época de coleta de sêmen, idade à coleta como covariável, efeitos linear e quadrático. Para o perímetro escrotal, foram incluídos os efeitos fixos de ano do nascimento, classe de idade à medição do perímetro e central de inseminação. Para idade ao primeiro parto, foram incluídos os efeitos fixos de rebanho-ano-estação de nascimento e os efeitos aleatórios de animal e residual. As herdabilidades para perímetro escrotal e idade ao primeiro parto foram, respectivamente, 0,37 e 0,22 . A tendência genética da idade ao primeiro parto foi significativa, com valor estimado de $-0,018$ mês/ano, e mostra que praticamente não houve progresso genético nessa característica ao longo dos anos estudados. As correlações genéticas obtidas em análises bicaracterísticas entre perímetro escrotal com volume, concentração, vigor, motilidade, defeitos maiores, menores e totais, número de doses, número total de espermatozoides viáveis e idade ao primeiro parto foram de 0,$33 ; 0,22 ; 0,91 ; 0,86 ;-0,07 ;-0,03 ;-0,04 ; 0,30 ; 0,23$ e -0,37, respectivamente. Esses resultados sugerem melhorias na eficiência reprodutiva de fêmeas quando utilizados nos rebanhos touros com maior perímetro escrotal.

Palavras-chave: correlação genética, idade ao primeiro parto, perímetro escrotal, qualidade do sêmen, tendência genética

\section{Genetic parameters for reproductive traits of dairy Gyr breed males and females}

\begin{abstract}
This work aimed to estimate genetic parameters for age at first calving, scrotal circumference, and seminal traits, and to evaluate genetic trend for age at first calving for dairy Gyr breed animals by analyzing data of 7,055 females and 97 males from several Brazilian herds. Covariance components were estimated by using the restricted maximum likelihood method under animal model in univariate analyses. The model for seminal traits included the company-year-season of semen collection fixed effects, age at the collection as a covariate, linear and quadratic effects. For scrotal circumference, it was included year of birth, age class at measurement, and insemination company fixed effects. For age at first calving, it was included herd-year-season of birth fixed effects and animal and residual random effects. The heritabilities for scrotal circumference and age at first calving were, respectively, 0.37 and 0.22 . The genetic trend of age at first calving was significant, with estimated value of -0.018 month/year and it shows that genetic progress in this trait practically did not occur over the studied years. The genetic correlations from bivariate analyses among scrotal circumference with volume, concentration, vigor, motility, major, minor, and total defects, number of doses, total number of feasible spermatozoid and age at first calving were $0.33,0.22,0.91,0.86,-0.07,-0.03,-0.04,0.30,0.23$ and -0.37 , respectively. These results suggest improvements in reproductive efficiency of females when they are used in herds with bulls with greater scrotal circumference.
\end{abstract}

Key Words: age at first calving, genetic correlation, genetic trend, scrotal circumference, semen quality 


\section{Introdução}

A eficiência econômica e produtiva do sistema de gado de leite está diretamente ligada ao desempenho reprodutivo do rebanho. Com o aumento dos custos de produção, os produtores de leite têm procurado formas para melhorar o desempenho zootécnico de seus animais, bem como evitar gastos adicionais.

Dentre as características reprodutivas mais selecionadas em bovinos, principalmente de corte, estão o perímetro escrotal e a idade ao primeiro parto, o que se deve, em parte, às suas respectivas herdabilidades, facilidade de mensuração e relações favoráveis entre essas características (Gressler et al., 2000; Boligon et al., 2007; Lopes et al., 2008). O perímetro escrotal tem sido relatado também como um indicador da qualidade do sêmen dos touros (Bergmann et al., 1997; Silveira et al., 2010). Porém, pouco se sabe sobre as relações existentes entre as características do sêmen e as características reprodutivas nas fêmeas. A idade ao primeiro parto é uma das características de maior relevância para o desempenho reprodutivo dos bovinos, estando intimamente relacionada com a puberdade, taxa de crescimento, maturidade sexual e aparecimento do primeiro cio fértil (Pereira et al., 1991; Meirelles et al., 2009). A vaca que procria mais cedo fica menos ociosa no rebanho e tem maior vida reprodutiva e produtiva, diminuindo o número de novilhas em recria necessárias à reposição (Dias et al., 2004; Silva et al., 2005), o que é de extrema importância para os sistemas de produção de gado de leite.

Em termos de melhoramento genético de gado de leite, pouco tem sido estudado quanto às características reprodutivas. Algumas causas que restringem a seleção dessas características são a falta de estimativas de parâmetros genéticos e maior atenção às características produtivas.

O monitoramento da mudança genética das características reprodutivas nos rebanhos permite observar se os programas de seleção adotados pelos criadores têm sido favoráveis à melhoria genética. Dessa forma, torna-se necessário analisar a tendência genética ao longo do tempo para avaliar o progresso genético que vem sendo alcançado e, ainda, nortear os programas de melhoramento (Malhado et al., 2008).

Objetivou-se com este estudo estimar parâmetros genéticos para a idade ao primeiro parto, perímetro escrotal e características do sêmen e avaliar a tendência genética da idade ao primeiro parto para animais da raça Gir Leiteira.

\section{Material e Métodos}

As informações utilizadas são provenientes de medidas de perímetro escrotal e medidas de qualidade do sêmen de touros Gir Leiteira e idade ao primeiro parto de suas filhas puras. Os dados das fêmeas são parte do Arquivo Zootécnico Nacional (AZN) Gado de Leite, base de dados gerenciada pelo Centro Nacional de Pesquisa em Gado de Leite, Embrapa Gado de Leite. Os touros, em sua grande maioria, participaram ou participam do Programa Nacional de Melhoramento do Gir Leiteiro, projeto executado pela Embrapa Gado de Leite em parceria com a Associação Brasileira dos Criadores de Gir Leiteiro (ABCGIL).

Os touros tiveram sêmen coletado durante a permanência em uma das seguintes seis centrais particulares de coleta de sêmen: Bela Vista, CRV Lagoa, Sembra, ABS Pecplan, Nova Índia e Sersia. Em geral, cada touro teve seu sêmen coletado mais de uma vez, sendo as coletas realizadas sequencialmente de dois em dois dias. Em cada coleta, foram avaliados os aspectos físicos (volume, motilidade, vigor e concentração) e morfológicos (defeitos maiores e menores) do sêmen. O perímetro escrotal, medido uma única vez em cada touro, foi mesurado em centímetros com uma fita métrica milimetrada, na posição mediana do escroto, no ponto de maior dimensão.

Após a medição do volume do ejaculado (mL), amostras foram obtidas para determinar a concentração espermática (número de espermatozoides/mL), a motilidade (\%) e o vigor (escala de 1 a 5). Os espermatozoides foram avaliados quanto à porcentagem de defeitos maiores e menores utilizando-se as técnicas de contraste de fase e da lâmina corada. Os defeitos totais foram obtidos pela soma dos defeitos menores e defeitos maiores. Além dessas características, foi calculado o número total de espermatozoides, multiplicando-se o volume pela concentração, para assim se obter o número total de espermatozoides viáveis, multiplicando-se o número total de espermatozoides pela motilidade. O número de doses produzidas por touro em cada coleta também foi avaliado. Conforme Martinez et al. (2000), as características vigor e porcentagem de defeitos maiores, menores e totais foram transformadas para $\sqrt{\mathrm{y}+0,5}$, enquanto a motilidade dos espermatozoides, para arco seno de $\sqrt{\mathrm{y} / 100}$ para que 0 pressuposto de normalidade fosse obedecido. Foram realizadas análises para verificar a efetividade das transformações. Não se adotou transformação da característica vigor, já que não houve diferença entre os resultados obtidos com a variável não transformada e a transformada, procedimento semelhante ao adotado por Meyer et al. (1990) e Gressler et al. (2000). O número de espermatozóides vivos foi dividido por 1000, por sua grande magnitude, que impedia análises no programa utilizado nas análises genéticas. 
Na estimação de parâmetros genéticos para a característica idade ao primeiro parto, utilizaram-se informações de 7.055 vacas, nascidas entre 1979 a 2004, pertencentes a 222 rebanhos, do Sudeste, Centro-Oeste e Nordeste brasileiros, com a característica mensurada entre 1982 e 2007 e no máximo a 60 meses de idade. A matriz de parentesco, nesse caso, foi composta por 14.195 animais.

Para a estimação de parâmetros genéticos para perímetro escrotal, foram utilizadas informações de 97 touros, pertencentes a 27 rebanhos, coletadas entre os anos de 1986 e 2008, em animais de 18 a 48 meses de idade, nascidos entre os anos de 1983 a 2006. A matriz de parentesco incluiu, nesse caso, 1.120 animais.

As correlações entre as características do sêmen e idade ao primeiro parto e entre perímetro escrotal e idade ao primeiro parto foram estimadas considerando dados dos reprodutores que passaram pelo processo de coleta de sêmen há no máximo três meses da data em que se mediu o perímetro escrotal. A partir daí, verificou-se que havia no máximo dez coletas de sêmen por touro e que estas eram homogêneas quanto às características do sêmen para cada reprodutor. Aplicou-se, então, a média aritmética sobre cada característica da qualidade do sêmen, obtendo, dessa forma, uma única medida de cada característica por animal. Os touros ainda deveriam ter ao menos três filhas com registro de primeiro parto. Ao final dessa edição, restaram informações referentes a 75 touros Gir Leiteiro, nascidos entre 1983 e 2001, e 803 filhas puras, nascidas entre 1988 e 2004, pertencentes a 75 rebanhos, com idade ao primeiro parto entre 26 e 60 meses, média de 42,23 meses. A matriz de parentesco foi composta, nesse caso, por 1.878 animais.

Para identificar quais efeitos dos fatores não-genéticos que influenciaram $(\mathrm{P}<0,05)$ as características a serem avaliadas, foi realizada análise prévia utilizando o método dos quadrados mínimos, pelo procedimento GLM(SAS, 2003).

As análises foram conduzidas sob modelo animal. O modelo estatístico para idade ao primeiro parto incluiu o efeito fixo de grupo contemporâneo, formado pela combinação rebanho (1 a 222), ano (1979 a 2004) e estação de nascimento (outubro a março = época 1 ; abril a setembro = época 2). Para as características de qualidade do sêmen, o modelo estatístico inclui os efeitos de grupo contemporâneo, formado pela combinação de central (1 a 6), ano(1986 a 2008) e época de coleta (outubro a março = época 1; abril a setembro = época 2), idade do animal à coleta como covariável, efeito linear e quadrático. Para perímetro escrotal, o modelo estatístico incluiu os efeitos fixos da central de coleta de sêmen (1, 2, 3, 4, 5 e 6), ano de nascimento (1983 a 2006), classe de idade à mensuração (18 a 24 meses $=1 ; 24$ a 30 meses $=2$; 30 a 36 meses $=3$; 36 a 48 meses $=4$ ). Todos os modelos incluíram o efeito aleatório do animal, com média 0 e variância $\sigma^{2}$ a e erro aleatório associado a cada observação, com média 0 e variância $\sigma^{2}{ }^{2}$.

Foram realizadas análises bicaracterísticas para a determinação das correlações genéticas entre as características da qualidade do sêmen e idade ao primeiro parto e entre perímetro escrotal e idade ao primeiro parto com os mesmos modelos das análises de características simples. O modelo para análises bicaracterísticas, em notação matricial, é descrito da seguinte maneira:

$\left[\begin{array}{l}y_{1} \\ y_{2}\end{array}\right]=\left[\begin{array}{cc}X_{1} & 0 \\ 0 & X_{2}\end{array}\right]\left[\begin{array}{l}\beta_{1} \\ \beta_{2}\end{array}\right]+\left[\begin{array}{cc}Z_{1} & 0 \\ 0 & Z_{2}\end{array}\right]\left[\begin{array}{l}u_{1} \\ u_{2}\end{array}\right]+\left[\begin{array}{l}\varepsilon_{1} \\ \varepsilon_{2}\end{array}\right]$,

em que $\mathrm{y}_{\mathrm{i}}=$ vetor de observações para a característica i; $\mathrm{X}_{\mathrm{i}}$ e $\mathrm{Z}_{\mathrm{i}}=$ matrizes de incidência relativas às observações da característica $\mathrm{i}$, para os efeitos fixos e de animal, respectivamente; $\beta_{i}=$ vetor de efeitos fixos para a característica i; $u_{i}=$ vetor de efeitos aleatórios de animal para a característica i; $\varepsilon_{i}=$ vetor de erros aleatórios para a característica i.

Os componentes de covariância utilizados para estimar os parâmetros genéticos para perímetro escrotal e idade ao primeiro parto foram obtidos pelo método da máxima verossimilhança restrita, com a utilização do aplicativo REMLF90 (Misztal, 2002). O critério de convergência utilizado foi de $10^{-11}$.

Foram obtidas estimativas das tendências genéticas para a característica idade ao primeiro parto, por meio da análise de regressão ponderada pelo número de observações, utilizando-se o procedimento REG (SAS, 2003). A variável dependente foram os valores genéticos para idade ao primeiro parto, enquanto a variável independente foi o ano de nascimento das vacas.

\section{Resultados e Discussão}

Em geral, as características da qualidade do sêmen (Tabela 1) apresentaram-se dentro dos padrões desejáveis, preconizados pelo Colégio Brasileiro de Reprodução Animal (CBRA, 1998). Bergmann et al. (1997) e Silva et al. (2002), trabalhando com dados de animais da raça Nelore com idade aproximada de 36 meses, também encontram valores semelhantes para as características aqui estudadas, com exceção do volume do ejaculado (entre 3,5 e 4 mL) e do perímetro escrotal (entre $33 \mathrm{~cm}$ e $36 \mathrm{~cm}$ ), que tenderam a ser maiores neste estudo. Os valores encontrados foram próximos aos obtidos por Martinez et al. (2000) para animais Gir. 
Tabela 1 - Padrão de qualidade do sêmen de touros doadores, segundo o Colégio Brasileiro de Reprodução Animal (CBRA, 1998)

\begin{tabular}{|c|c|c|c|c|}
\hline Característica $^{1}$ & Número de observações & Média & Desvio-padrão & Padrão CBRA \\
\hline Volume (mL) & 195 & 5,13 & 2,70 & $\geq 0,25$ \\
\hline $\mathrm{MVOL}^{2}$ & 75 & 6,55 & 2,57 & - \\
\hline Concentração (espermatozoides/mL) & 195 & 1.152 & 733 & - \\
\hline MCONC $^{2}$ & 75 & 1.361 & 466 & - \\
\hline Vigor (1 a 5) & 179 & 3,72 & 0,60 & $\geq 3$ \\
\hline $\mathrm{MVIG}^{2}$ & 75 & 3,5 & 1,02 & - \\
\hline Motilidade (\%) & 195 & 62,89 & 9,35 & $\geq 30$ \\
\hline $\mathrm{MMOT}^{3}$ & 75 & 0,86 & 0,12 & - \\
\hline Defeitos maiores (\%) & 79 & 7,79 & 3,33 & $\leq 20$ \\
\hline MDMA $^{3}$ & 75 & 1,82 & 1,30 & - \\
\hline DME (\%) & 79 & 5,33 & 3,54 & - \\
\hline $\mathrm{MDME}^{3}$ & 75 & 1,54 & 1,18 & - \\
\hline Defeitos totais (\%) & 79 & 13,12 & 5,26 & $\leq 30$ \\
\hline $\mathrm{MDT}^{3}$ & 75 & 3,37 & 2,40 & - \\
\hline Doses ( $\left.\mathrm{n}^{\mathrm{o}}\right)$ & 160 & 132,64 & 74,81 & - \\
\hline MNDOSE $^{2}$ & 75 & 113,14 & 96,26 & - \\
\hline Perímetro escrotal (cm) & 97 & 36,05 & 2,62 & - \\
\hline Idade ao primeiro parto (meses) & 7.055 & 43,08 & 7,38 & - \\
\hline
\end{tabular}

${ }^{1}$ VOL = volume CONC = concentração VIG = vigor MOT = motilidade DMA = defeitos maiores DME = defeitos menores; DT = defeitos totais NDOSE = número de doses.

2 Média da variável não transformada.

${ }^{3}$ Média da variável transformada.

A média de idade ao primeiro parto foi mais alta quando comparada aos valores obtidos em trabalhos com animais de corte. Mercadante et al. (2000) obtiveram média de 38,3 meses para animais Nelore, enquanto Dias et al. (2004) encontraram média de 34 meses para idade ao primeiro parto. A superioridade dos animais de corte em relação aos deste estudo pode estar relacionada às associações favoráveis entre características de desenvolvimento corporal e a idade ao primeiro parto.

As herdabilidades, variâncias genéticas e residuais para perímetro escrotal e idade ao primeiro parto, obtidas em análises uni-característica, foram respectivamente 0,37, 2,46, 4,12 e 0,22, 6,69, 23,24. A herdabilidade da idade ao primeiro parto para as vacas Gir Leiteiro, foi inferior a estimativa de herdabilidade $(0,56)$ encontrada por Wenceslau et al. (2000) para a mesma raça, porém com volume de dados muito reduzido. A estimativa obtida neste trabalho foi semelhante às encontradas na literatura para animais das raças Nelore (entre 0,08 e 0,28) (Mercadante et al., 2000; Pereira et al., 2000; Dias et al., 2004), Guernsey, 0,13 (Hermas, et al., 1987) e Holandesa, 0,19 (Cerón-Muñoz et al., 2004).

A idade ao primeiro parto, o perímetro escrotal e as características de qualidade do sêmen (Tabela 2) foram, em geral, correlacionadas favoravelmente com a idade ao primeiro parto, com exceção dos defeitos menores $(-0,12)$. Contudo, essa correlação foi de pequena magnitude. Os defeitos maiores e totais apresentaram correlações positivas, porém baixas, com a idade ao primeiro parto, logo touros que produzem sêmen com maiores defeitos produzem progênies com maiores idades ao primeiro parto. A concentração, a motilidade e o vigor foram negativamente correlacionados com a idade ao primeiro parto, de magnitude alta $-0,98)$, média $(-0,33)$ e baixa $(-0,19)$ respectivamente. Assim, a seleção de touros que produzam sêmen de maior qualidade pode trazer, como resposta correlacionada, menor idade ao primeiro parto de suas filhas. A literatura é escassa quanto a tais correlações.

O perímetro escrotal foi correlacionado favoravelmente com a idade ao primeiro parto, o que também foi verificado por diversos autores em trabalhos com animais de corte. Ressalta-se, no entanto, que o resultado deste estudo foi superior aos obtidos para a raça Nelore. Boligon et al. (2007) encontraram estimativas de correlações genéticas entre o perímetro escrotal medido aos 12 e 18 meses de idade e a idade ao primeiro parto das fêmeas, respectivamente, de $-0,13$ e $-0,23$, demonstrando que a seleção de reprodutores para maior perímetro escrotal poderá trazer, como resposta correlacionada, menor idade ao primeiro parto de suas progênies. Pereira et al. (2000) encontraram estimativa de $-0,22$, entre o perímetro escrotal aos 18 meses de idade e a idade ao primeiro parto das fêmeas. Eles concluíram que essa correlação permite a utilização do perímetro escrotal como critério de seleção para melhorar a eficiência reprodutiva das fêmeas. Semelhantemente ao presente estudo, Martínez-Velázquez et al. (2003) obtiveram estimativas de herdabilidade de idade ao primeiro parto $(0,16)$ e perímetro escrotal $(0,41)$ e correlação genética 
Tabela 2 - Correlações genéticas entre as características reprodutivas obtidas em análises bicaracterísticas

\begin{tabular}{|c|c|c|c|c|c|c|c|c|c|}
\hline Característica $^{1}$ & \multicolumn{9}{|c|}{ Correlação genética } \\
\hline Idade ao primeiro parto & $-0,37$ & 0,27 & $-0,98$ & $-0,19$ & $-0,33$ & 0,26 & $-0,12$ & 0,10 & $-0,46$ \\
\hline
\end{tabular}

entre essas características de -0,26, para animais de nove raças europeias. Essa mesma relação também foi confirmada por Vargas et al. (1998) ao trabalharem com a raça Brahman.

As correlações observadas entre as características reprodutivas de machos e fêmeas podem, em parte, ser devidas ao desenvolvimento corporal do reprodutor. Assim, um touro de melhor desenvolvimento corporal pode transmitir às suas filhas tal característica e, desta forma, favorecer características reprodutivas como a idade ao primeiro parto, que são muito relacionadas ao peso do animal. Em sistemas de produção de gado de leite, é comum a adoção de uma idade padrão para se poder inseminar ou cobrir uma novilha, ignorando-se em muitos casos o desenvolvimento corporal dela, fato que pode influenciar negativamente as correlações entre o perímetro escrotal e a idade ao primeiro parto.

A tendência genética é uma medida que permite avaliar a mudança ocasionada por um processo de seleção em determinada característica ao longo dos anos. A tendência genética na raça Gir Leiteira foi significativa $(\mathrm{P}<0,01)$ e os resultados de avaliação mostraram ganho acumulado na idade ao primeiro parto de -0,3 mês ao longo de 17 anos (Figura 1). O ganho por ano foi de $-0,018$ mês, portanto, praticamente não houve progresso genético nessa característica no período estudado.

Considerando as estimativas dos parâmetros genéticos, o ganho genético anual esperado na idade ao primeiro parto quando a seleção é praticada no perímetro escrotal em intervalo de geração de 57 meses e quando $10 \%$ dos machos são selecionados para a reprodução, a resposta à seleção, é-0,2878 mês. Entretanto, considerando a idade ao primeiro parto de 43 meses (média da raça Gir Leiteira), supondo, nesse caso, intervalo de geração de 86 meses e proporção de fêmeas selecionadas de $60 \%$, a resposta à seleção direta nesta característica alcança $37 \%$ da resposta correlacionada.

De acordo com as correlações encontradas entre o perímetro escrotal e a idade ao primeiro parto, suas herdabilidades e a resposta à seleção, parece ser mais vantajosa a seleção para maior perímetro escrotal nos machos do que propriamente para a menor idade ao primeiro parto nas fêmeas. O perímetro escrotal ainda possui a vantagem

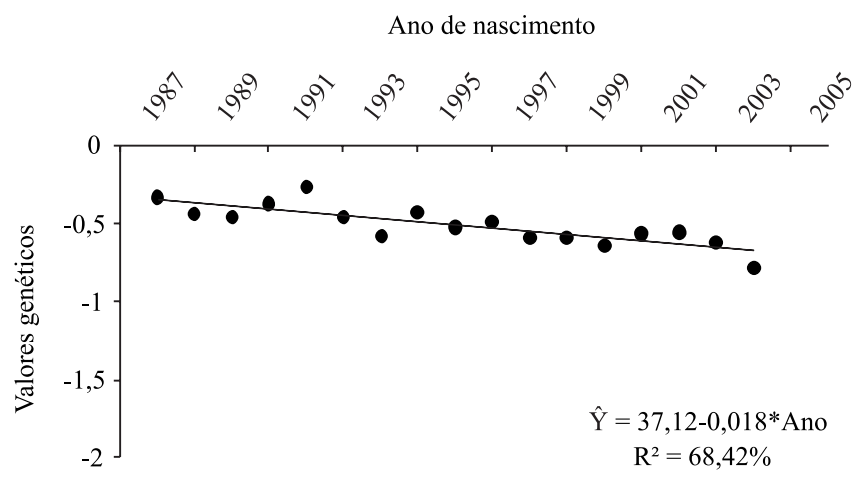

Figura 1 - Tendência genética da idade ao primeiro parto, em meses, na raça Gir Leiteira considerando o ano de nascimento.

de ser facilmente mensurado, e essa mensuração pode ser feita mais cedo na vida do macho do que a idade ao primeiro parto na vida da fêmea. Logo, a medição do perímetro escrotal poderia ser realizada assim que o tourinho fosse inscrito no teste de progênie.

A utilização do perímetro escrotal como critério de seleção para melhorar a eficiência reprodutiva das progênies poderia ser feita enquanto não se obtêm informações necessárias para a geração da capacidade prevista de produção para a idade ao primeiro parto de cada touro participante do teste de progênie da raça.

Contudo, as associações entre características de machos e fêmeas devem ser vistas com cautela. Mais estudos nesse sentido devem ser realizados a fim de confirmar os resultados para a raça Gir leiteira. O perímetro escrotal dos reprodutores pode, a princípio, ser utilizado com a finalidade de auxiliar a seleção para menores idades ao primeiro parto das progênies. É necessária, ainda, a determinação da melhor idade para se medir o perímetro escrotal nos reprodutores Gir Leiteiros mediante a avaliação de qual a idade está mais correlacionada geneticamente com as características reprodutivas de suas progênies e as possíveis influências da condição corporal dos reprodutores sobre essas características. 


\section{Conclusões}

Os parâmetros genéticos obtidos para as características da qualidade do sêmen, idade ao primeiro parto e perímetro escrotal possibilitam obter ganhos genéticos satisfatórios em programas de melhoramento genético. As correlações entre perímetro escrotal e idade ao primeiro parto permitem, ainda, a utilização do perímetro escrotal como critério de seleção para melhorar a eficiência reprodutiva nas fêmeas, assim, sugere-se a inclusão do perímetro escrotal nas avaliações genéticas de reprodutores da raça Gir Leiteira. Praticamente não houve progresso genético na idade ao primeiro parto na raça ao longo dos anos estudados, porém existe variabilidade genética suficiente para promover progresso genético pela seleção da idade ao primeiro parto.

\section{Agradecimentos}

Às centrais de inseminação artificial Bela Vista, CRV Lagoa, Sembra, ABS Pecplan, Nova Índia e Sersia por gentilmente cederem os dados para a realização deste estudo.

\section{Referências}

BERGMANN, J.A.G.; QUIRINO, C.R.; VALE FILHO, V.R. et al. Herdabilidades e correlações genéticas entre medições testiculares e características espermáticas em touros Nelore. Archivos Latinoamericanos de Producción Animal, v.5, n.1, p.473-475, 1997.

BOLIGON, A.A.; RORATO, P.R.N.; AlBUQUERQUE, L.G. Correlações genéticas entre medidas de perímetro escrotal e características produtivas e reprodutivas de fêmeas da raça Nelore. Revista Brasileira de Zootecnia, v.36, n.3, p.565-571, 2007.

CERÓN-MUÑOZ, M.F.; TONHATI, H.; COSTA, C.N. et al. Genotype $\times$ environment interaction for age at first calving in Brazilian and Colombian Holsteins. Journal of Dairy Science, v.87, p.2455-2458, 2004

COLÉGIO BRASILEIRO DE REPRODUÇÃO ANIMAL - CBRA. Manual para exame andrológico e avaliação de sêmen animal. 2.ed. Belo Horizonte: 1998. 49p.

DIAS, L.T.; EL FARO, L.; ALBUQUERQUE, L.G. Estimativas de herdabilidade para idade ao primeiro parto de novilhas da raça Nelore. Revista Brasileira de Zootecnia, v.33, n.1, p.97-102, 2004.

GRESSLER, S.L.; BERGMANN, J.A.G.; PEREIRA C.S. et al. Estudo das associações genéticas entre perímetro escrotal e características reprodutivas de fêmeas Nelore. Revista Brasileira de Zootecnia, v.29, n.2, p.427-437, 2000.

HERMAS, S.A.; YOUNG, C.W.; RUST, J.W. Genetic relationships and additive genetic variation of productive and reproductive traits in Guernsey dairy cattle. Journal of Dairy Science, v.70, p.1252-1257, 1987.
LOPES, C.R.A.; BARBOSA, S.B.P.; PEREIRA, R.G.A. et al Eficiência reprodutiva e influência de fatores de meio e de herança sobre a variação no peso ao nascer de bubalinos no estado de Rondônia. Revista Brasileira de Zootecnia, v.37, n.9, p.1595-1600, 2008.

MALHADO, C.H.M.; CARNEIRO, P.L.S.; PEREIRA, D.G. et al. Progresso genético e estrutura populacional do rebanho Nelore no Estado da Bahia. Pesquisa Agropecuária Brasileira, v.43, n.9, p.1163-1169, 2008.

MARTINEZ, M.L.; VERNEQUE, R.S.; TEODORO, R.L. et al. Correlações entre características da qualidade do sêmen e a circunferência escrotal de reprodutores da raça Gir. Revista Brasileira de Zootecnia, v.29, n.3, p.700-706, 2000.

MARTÍNEZ-VELÁZQUEZ, G.; GREGORY, K.E.; BENNETT, G.L. Genetic relationships between scrotal circumference and female reproductive traits. Journal of Animal Science, v.81, p.395-401, 2003.

MEIRELLES, S.L.; ESPASANDIN, A.C.; MATTAR, M. et al. Genetic and environmental effects on sexual precocity traits in Nellore cattle. Revista Brasileira de Zootecnia, v.38, n.8, p.1488-1493, 2009.

MERCADANTE, M.E.Z.; LOBO, R.B.; OLIVEIRA, H.N. Estimativas de (Co)variâncias entre características de reprodução e de crescimento em fêmeas de um rebanho Nelore. Revista Brasileira de Zootecnia, v.29, n.4, p. 997-1004, 2000.

MEYER, K.; HAMMOND, K.; PAMELL, P.F. et al. Estimates of heritability and repeatability for reproductive traits in Australian beef cattle. Livestock Production Science, v.25, n.1, p.15-30, 1990.

MISZTAL, I. REMLF90 manual. [2002]. Disponível em: <http:// nce.ads.uga.edu/ ignacy/numpub/blupf90/docs/remlf90.pdf $>$ Acesso em: 27/11/2008.

PEREIRA, J.C.C.; AYALA, J.M.N.; OLIVEIRA, H.N. Efeitos genéticos e não-genéticos sobre a idade ao primeiro parto e o intervalo entre partos de duas populações da raça Nelore. Arquivo Brasileiro de Medicina Veterinária e Zootecnia, v.43, n.1, p.93-102, 1991.

PEREIRA, E.; ELER, J.P.; FERRAZ, J.B.S. Correlação genética entre perímetro escrotal e algumas características reprodutivas na raça Nelore. Revista Brasileira de Zootecnia, v.29, n.6, p.1676-1683, 2000.

STATISTICAL ANALYSIS SYSTEM - SAS. User's guide. Version 9. 1.ed. Cary: SAS Institute, 2003. 5136p..

SILVA, A.E.D.F.; UNANIAN, M.M.; CORDEIRO, C.M.T. et al. Relação da circunferência escrotal e parâmetros da qualidade do sêmen em touros da raça Nelore, PO. Revista Brasileira de Zootecnia, v.31, n.3, p.1157-1165, 2002.

SILVA, M.D.; BARCELlOS, J.O.J.; PRATES, E.R. Desempenho reprodutivo de novilhas de corte acasaladas aos 18 ou aos 24 meses de idade. Revista Brasileira de Zootecnia, v.34, n.6, p.2057-2063, 2005.

SILVEIRA, T.S.; SIQUEIRA, J.B.; GUIMARÃES, S.E.F. et al. Maturação sexual e parâmetros reprodutivos em touros da raça Nelore criados em sistema extensivo. Revista Brasileira de Zootecnia, v.39, n.3, p.503-511, 2010.

VARGAS, C.A.; ELZO, M.A.; CHASE, C.C. et al. Estimation of genetic parameters for scrotal circumference, age at puberty in heifers, and hip height in Brahman cattle. Journal of Animal Science, v.76, p.2536-2541, 1998.

WENCESLAU, A.A.; LOPES, P.S.; TEODORO, R.L. et al. Estimação de parâmetros genéticos de medidas de conformação, produção de leite e idade ao primeiro parto em vacas da raça Gir leiteiro. Revista Brasileira de Zootecnia, v.29, n.1, p.153-158, 2000 . 